\title{
New sources of sex cells
}

\author{
Within the next decade or so, it will become possible to derive sperm and eggs from skin cells. The ethical \\ and technical hurdles need to be addressed with the scientific and therapeutic benefits in mind.
}

- arlier this month, the world had its first look at a pregnant man,

- a jarring reminder of how conventions in the way humans are _ created could shift. Just a week later, scientists, bioethicists, lawyers and journal editors convened in Hinxton, UK, to ponder how long it will be until sperm and eggs can be made entirely in a Petri dish from, say, skin cells induced to pluripotency. They asked questions such as how will these advances transform reproductive research and medicine? How might they change society? Is the bioethics community prepared?

These questions have acquired a sense of urgency, at least in Britain, where a government bill updating the 1990 Human Fertilisation and Embryology Act is working its way through the system. If passed by the House of Commons in its present form, the bill would allow basic research on human gametes derived in vitro but would not permit the use of such gametes for fertility treatment. Opponents may yet propose amendments designed to stop all embryo research - including the demonstration that in-vitro-derived gametes are normal by seeing whether they are capable of fertilization and further development. The Hinxton group's consensus statement, arrived at after three days of discussion, can be viewed at www.hinxtongroup.org/Consensus_HG08_FINAL.pdf.

The organizers scheduled the topic of pluripotent stem cell derived gametes (PSCDGs) two years ago. Since then, making induced pluripotent stem (iPS) cells has become relatively straightforward, but although the technique circumvents the ethical and technical problems associated with collecting human eggs and creating embryos, there are still serious ethical implications. Indeed, the facility with which iPS cells can be derived could make it easier to derive gametes from any person, living or dead.

The potential benefits of PSCDGs are impressive. They could reveal much about the mechanism of gamete development, with implications for treating infertility, certain cancers and genetic diseases, and for facilitating drug tests on gametes. Other potential applications present social and ethical challenges: germline genetic modification for the correction of disease mutations or for research; various forms of biological enhancement; genetic screening of embryos for selection; and enabling same-sex couples to have their own genetic offspring.

The Hinxton group believes that eggs and sperm will be generated from pluripotent stem cells in 5-15 years, although it concedes this is something of a guess. Technically, a major sticking point is getting the developing gametes to undergo meiosis. Ethically, the problem is quality control: the only conclusive way to test that gametes are functional and can create a viable embryo is to go ahead and do so.

Once gametes can be made, the immediate question will be whether and how embryos derived from them can be created and studied. So iPS research will come full circle, and we will be faced again with the issue that has dogged us since the birth of Louise Brown, the first IVF baby in 1978. Is it moral to use human embryos for research or to create human life with the implicit intention of destroying it during an experiment?

Not conducting this research could cause harm: without it, a pregnancy using an embryo produced by PSCDGs would pose an unacceptable risk for both child and mother. If PSCDGs are to one day join the tools of assisted reproduction, functional tests must be developed to eliminate faulty gametes. Quality tests developed for IVF will be useful in this context and the methods should first be exhaustively assessed on animal models, including non-human primates.

Clearly, appropriate oversight of PSCDG research is needed, but much of the framework for doing so is already in place, in the form of committees for overseeing stem-cell research and institutional review boards for human experimental subjects. Nonetheless, 14 countries already prohibit or restrict PSCDG technology for genetic screening or germline genetic modification, some under penalty of imprisonment. Japan prohibits the creation of human PSCDGs altogether. Where stricter regulations are needed in many countries is on experimental fertility treatments involving human embryos carried out in clinics. But for any country considering legislation in this area, beware of impeding basic research and losing the benefits that could result.

\section{The big ome}

\section{It's time to make the case for proteins.}

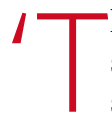

he body's building blocks,' 'cellular machinery': proteins are sometimes stuck with rather mundane labels. Certainly they sit in the scientific shadow of the genome sequence and the eulogies that inspires. So protein biologists face an uphill battle if they are to fire up the research community - and the world beyond enough to buy into a Human Proteome Project.
The project is just getting past the back-of-the-envelope stage but, in essence, it would systematically catalogue all the proteins manufactured in the body: what they are, where they are and in what abundance (see page 920). A cancer biologist might reveal whether a rogue protein is overexpressed in the tumours she studies compared with levels from healthy tissue that are logged in the proteome register. A geneticist who traces Alzheimer's susceptibility to a region of code could consult the proteome to reveal which proteins are being manufactured from that region in the brain. We can expect this catalogue of proteins to eventually include the targets for almost all future drugs.

There are many obvious parallels with efforts to elucidate the 